\title{
Abordaje Contrafáctico, Inferencia Causal y el Enfoque de la Puntuación de la Propensión. Efectos de las Remesas en la Escolarización Paraguaya
}

\section{Counterfactual Approach, Causal Inference and Propensity Score Analysis Effects of Remittances on Paraguayan Schooling}

\author{
Pablo Sebastián Gómez \\ Universidad Nacional de Córdoba (UNC). \\ Consejo Nacional de Investigaciones Científicas y Técnicas (CONICET-CIECS). \\ Argentina. \\ enclavepablo@yahoo.com.ar(ARGENTINA)
}

Recibido: 31.072012

Aceptado: 24.03.2014

\section{RESUMEN}

La mayoría de la investigación en ciencias sociales en general y en el campo de los estudios migratorios en particular, está confrontada con el problema de la causalidad cuando evalúa el impacto de alguna variable de interés. El abordaje contrafáctico permite solucionar los problemas derivados de la asignación no aleatoria de las poblaciones bajo estudio a los grupos de control y tratamiento. En este artículo presentamos los problemas de analizar los efectos causales de las remesas provenientes del Sur Global en Paraguay, proponemos una solución y estimamos los efectos en la escolarización paraguaya. Los principales resultados obtenidos sugieren que no hay efectos en la inversión en capital humano y las potencialidades del enfoque de la puntuación de la propensión. 


\title{
PALABRAS CLAVE
}

Migración Sur-Sur, Sesgo de Selección, Causalidad, Puntuación de la Propensión, Remesas.

\begin{abstract}
Most research in social science in general, and in the field of migration studies, in particular, encounters the problem of causality when assessing the impact of any variable of interest. The counterfactual approach solves the problems arising from non-random allocation of the populations under study to control and treatment groups. In this paper, we present the problems of analyzing the causal effects of remittances from the Global South in Paraguay, we propose a solution and estimates the impact on Paraguayan schooling. The main results highlight that there are no effects on investment in human capital and the potential of the propensity score analysis.
\end{abstract}

\section{KEY WORDS}

South-South Migration, Selection Bias, Causality, Propensity Score, Remittances

\section{INTRODUCCIÓN}

Uno de los nudos gordianos de las ciencias sociales se encuentra en el problema de la explicación causal. La pregunta fundamental, todavía sigue en debate: ¿cuándo podemos establecer relaciones causales entre dos fenómenos sociales?. Claramente, hay diferentes tradiciones teóricas para responder a esta pregunta. Uno de los posibles caminos de solución es el abordaje contrafáctico. En términos generales, la premisa adoptada es la comparación entre "mundos similares" excepto en la variable causal a analizar; cuando se trabaja con datos que provienen de observaciones. Esta alternativa se presenta porque la mayoría de los científicos sociales no suelen utilizar el "estándar de oro" de la causalidad: el diseño de experimentos aleatorios. Los desafíos de utilizar datos observacionales para justificar afirmaciones causales son considerables (Winship y Morgan 1999; Shadish, Cook, y Campbell 2001; Goldthorpe 2001; Smith 2003; Winship y Sobel 2009; Rosenbaum 2009; Gangl 2010) y el abordaje contrafáctico es una alternativa de solución. Con origen en la estadística y en la literatura econométrica, el enfoque contrafáctico, Rubin, o modelo de resultados potenciales, en las últimas tres décadas se ha convertido en la herramienta conceptual estándar que unifica la noción de causalidad, permite entender el problema de la identificación en el centro de la inferencia causal y evaluar la utilidad de técnicas de estimación alternativas (Gangl 2010). 
En el campo de los estudios migratorios la discusión sobre la causalidad se desarrolló en relación al análisis del impacto de las remesas. En esa línea, uno de los problemas que más atención recibió fue el de las posibilidades de comparación entre conjuntos de hogares receptores y no-receptores de remesas. Estos problemas no son exclusivos de nuestros interrogantes; por el contrario, se presentan cada vez que es necesario comparar dos grupos que no han sido conformados aleatoriamente, $\mathrm{y}$ aparecen porque la pertenencia a cada grupo está determinada por factores que no suelen ser inmediatamente reconocibles. Los hogares receptores de remesas o que tienen miembros migrantes no son una muestra aleatoria de la población de origen. Esta asignación no aleatoria de las unidades de análisis a los grupos suele conocerse como sesgo en la selección o también auto-selección. La búsqueda de solución al problema de la auto-selección consiste en emular condiciones experimentales a partir de datos observacionales, y esto se aproxima constituyendo grupos tan semejantes como sea posible, excepto por la presencia de la variable cuyo impacto interesa observar. En estas condiciones, dado que no se cumplen los supuestos del diseño experimental, se busca simular una situación donde el grupo de control esté presente.

El dominante problema de la auto-selección es lo que hace necesaria la utilización de técnicas que puedan dar cuenta del mismo. En el campo de los estudios migratorios el tópico ha merecido trabajos destinados a lidiar con el problema, tanto en lugares de origen como en lugares de destino (Borjas 1987; Borjas 1990; Constant y Massey 2003; Chiswick 1999; Coulon y Piracha 2005; Chiquiar y Hanson 2005; Akee 2010; Bertoli 2010).

El objetivo general de este artículo es presentar el abordaje contrafáctico en su dimensión conceptual y su aplicación práctica a través del enfoque de la puntuación de la propensión. De manera específica se analizan los problemas derivados de investigar los efectos causales de las remesas en diversos componentes de la escolarización en Paraguay. El artículo está organizado en siete secciones. En la sección 2 se presenta y discute el problema del sesgo de selección. En la sección 3 nos aproximamos el abordaje contrafáctico. En la sección 4 se especifica la técnica de la puntuación de la propensión. En la sección 5 se aplica el abordaje contrafáctico al estudio del impacto de las remesas en áreas de origen de la migración, se utiliza el paquete MatchIt (Ho et al. 2007; Ho et al. 2011) del software R. En la sección 6 se discuten los resultados. Finalmente, en la sección 7 se presentan las conclusiones.

\section{EL PROBLEMA DEL SESGO DE SELECCIÓN}

Cuando analizamos efectos causales el problema fundamental está en el grupo de control para comparar o estimar los resultados que habrían ocurrido sin la migración o las remesas. Esto es así, porque hay ciertas características que son inobservables para el investigador (por ejemplo la capacidad de emprendedor) y que sin embargo son determinantes importantes en los resultados 
(Funkhouser 2006). Uno de los objetivos de este trabajo es establecer relaciones causales entre un factor (recepción de remesas) y una variable de interés, es decir, analizar cómo una característica de los hogares en la población (dimensiones de la escolarización) responde o cambia cuando el factor es introducido. En la fuente de datos utilizada, la Encuesta Permanente de Hogares de Paraguay, tenemos información sobre dos condiciones: hogares expuestos al factor (con remesas) y hogares no expuestos (sin remesas). Cuando trabajamos con efectos causales, el problema fundamental es si los grupos son suficientemente similares para que la comparación pueda ser interpretada como un efecto causal del factor (Weisberg 2010).

El problema derivado de comparar grupos que no son suficientemente similares ocurre por el sesgo de selección implícito en los hogares receptores de remesas. Este sesgo puede ser originado por una selección en la muestra o por auto-selectividad (endogeneidad)(Heckman 1979). El sesgo originado por una selección en la muestra supone una selección no aleatoria de ciertos individuos, basada en la disponibilidad de datos observables, como los ingresos salariales o la finalización del grado universitario. Según Titus (2007), en un estudio sobre el efecto del grado universitario en el ingreso salarial, si analizamos la muestra restringida solo a los individuos que reportan su salario, se pueden producir tasas sesgadas. Esto es así porque tal muestra está restringida y solo incluye aquellos individuos empleados.

Los problemas de la auto-selectividad ocurren cuando, continuando con el ejemplo anterior, el hecho de que el individuo tenga un grado universitario, es utilizado como predictor de su salario entre individuos empleados, y la naturaleza no-aleatoria de completar ese grado universitario es ignorada (Titus 2007).

En relación a la auto-selectividad, Chiswick (1999) señala que los migrantes frecuentemente son descriptos como diferentes de aquellos que no migraron en lugares de origen. Estos son vistos como más "agresivos", avezados, emprendedores. Así, una de las proposiciones aceptadas es que los migrantes están "auto-seleccionados" sobre la base de habilidades, fortalezas y otras características: es decir, serían más ambiciosos, emprendedores, etc. que aquellos individuos que optaron por quedarse en los lugares de origen. Sobre esta base, las familias receptoras de remesas -y con migrantes en general- tendrían particularidades específicas que hacen difícil una simple y directa comparación con los hogares no vinculados a la migración. Esta "auto-selección" tiene consecuencias en el análisis tanto para las regiones de origen como para las regiones de destino.

En términos metodológicos, la dificultad está en identificar el impacto causal de una variable $X_{t}$ sobre $y$ en datos observacionales, donde $X_{t}$ no es manipulable por el investigador y no puede asignarse aleatoriamente (Nichols 2007). Para Nichols (2007), estimar el impacto del "efecto del tratamiento" es

${ }^{1}$ Esto se aplica según Chiswick (1999) fundamentalmente a los migrantes por razones económicas

EMPIRIA. Revista de Metodología de Ciencias Sociales. N. ${ }^{\circ}$ 28, mayo-agosto, 2014, pp. 107-128. ISSN: 1139-5737, DOI: 10.5944/empiria.28.12123 
el objetivo de numerosos estudios en el campo de las ciencias sociales, inclusive cuando explícitamente los resultados sean propuestos en términos de asociación y no de efectos causales. La variable $X_{t}$ es definida como el "tratamiento", los diseños de investigaciones experimentales ofrecen las estimaciones menos sesgadas, pero estos experimentos frecuentemente son inviables en el campo de las ciencias sociales, tanto por cuestiones éticas como por el costo. Los diseños cuasi-experimentales ofrecen perspectivas que permiten trabajar con inferencias causales utilizando datos observacionales (Nichols 2007). Ante el dominante problema de la auto-selectividad en diversos fenómenos sociales, McKenzie y Sasin (2007) proponen cuatro alternativas metodológicas:

1. Experimentos aleatorios, creando "grupos de control" de individuos con las mismas características de los migrantes pero sin la opción de migrar. Por ejemplo ,McKenzie et al., (2010) analizan el caso de Nueva Zelanda y Tonga. Cada año Nueva Zelanda permite una cuota de migrantes de Tonga con un sistema al azar entre los postulantes excedentes. El trabajo de los autores permite estimaciones experimentales de ganancia de ingresos de los migrantes. Se compara los ingresos de los migrantes que postularon para migrar pero que no fueron seleccionados con el de los que postularon y fueron seleccionados. Además el ingreso con individuos que no aplicaron. Al comparar con este último grupo, mediante técnicas no experimentales, se permite evaluar el grado en el cual métodos no experimentales pueden proveer de estimaciones sin sesgo de la ganancia en el ingreso por la migración.

2. Ignorar la causalidad y trabajar con cruce de variables. Cruces de variables entre remesas y alguna variable de interés puede ser productivo. Tabulando las remesas por deciles de ingresos, puede ser productivo deducir las remesas del total del ingreso. Sin embargo, estos métodos no identifican relaciones causales.

3. Trabajar con regresión por mínimos cuadrados. El abordaje común es a través de regresiones de la variable de interés sobre la variable de migración y una serie de variables de control: Resultado $=\alpha+$ $\beta^{*}$ migración $+\gamma^{*} X+\varepsilon$. Se asume que a algunos hogares la migración o las remesas le "ocurren" (como un maná que cae del cielo). Sin embargo, aquí se ignora la endogeneidad y el coeficiente $\bigotimes$ esta sesgado. La fuente del sesgo radica en la violación del supuesto de independencia de la variable explicativa (migración) del término de error en la ecuación. Se puede aplicar el modelo de selección de Heckman para tratar de corregir por algún posible sesgo de selección. Sin embargo, tales métodos requieren la identificación de una variable instrumental (lo cual no siempre es simple). Consideremos el siguiente modelo de regresión por mínimos cuadrados: $\mathrm{Y}_{i}=\alpha+\tau W_{i}+X^{\prime}{ }_{i} \beta+\varepsilon_{i}$, donde $\mathrm{w}_{\mathrm{i}}$ es una variable dicotómica indicando el tratamiento, y $X_{\mathrm{i}}$ es el vector de variables independientes para el caso i. En datos observacionales, como el investigador no tiene control sobre la asignación a la condición de recepción de remesas, $\mathrm{W}$ está frecuentemente altamente correlacionado 
con $\mathrm{Y}$. Al respecto, el control estadístico es un proceso de modelado que intenta extraer la contribución independiente de las variables explicativas (ej: el vector $\mathrm{X}$ ) del resultado (Y) para determinar el efecto de $\tau$. Sin embargo, los supuestos estadísticos del modelo de mínimos cuadrados son violados cuando variables independientes relevantes son omitidas. Cuando tal omisión ocurre, las variables en $\mathrm{X}$ no pueden explicar todas las sistemáticas variaciones en $\mathrm{Y}$, y por lo tanto, el termino de error $\varepsilon$ está correlacionado con W (S. Guo, Barth, y Gibbons 2006). Las consecuencias de la correlación entre $\varepsilon$, y W conducen a estimaciones sesgadas e inconsistente de los efectos de las remesas.

4. Reconstruir el contrafáctico. Desarrollamos este punto.

\section{ABORDAJE CONTRAFÁCTICO PARA DATOS OBSERVACIONALES. LENGUAJE EXPERIMENTAL.}

El estado ideal para trabajar en términos de inferencia causal es a través del diseño experimental ${ }^{2}$, que asigna de manera aleatoria los individuos bajo estudio a un grupo de "control" y otro de "tratamiento" (Rosenbaum 2005; Rosenbaum 2009; Shadish, Cook, y Campbell 2001; Morgan y Winship 2007). De esta manera, los grupos son comparables bajo tratamientos diferentes. En estas circunstancias, que los grupos sean comparables antes del tratamiento asegura que las diferencias en los resultados después de la aplicación del mismo reflejen el efecto causal. Si una prueba estadística rechaza la hipótesis de que las diferencias se deben al azar, entonces el efecto del tratamiento es demostrado. Ho, Imai, King, \& Stuart (2007), señalan que los experimentos tienen tres características fundamentales: (1) selección aleatoria de las unidades que deben ser observadas en una población dada, (2) la asignación aleatoria de los valores del tratamiento en cada unidad observada, y (3) $\mathrm{N}$ grande (tamaño de la muestra).

Evidentemente, estas situaciones de experimentación con personas no son éticas o deseables y bajo tales circunstancias los efectos del tratamiento son analizados en "estudios observacionales" (Rosenbaum 2005). Definidos como una investigación empírica de los efectos causados por el tratamiento cuando la experimentación aleatoria no es deseable o carece de ética (Cochran 1965; Rosenbaum 2009). En esta perspectiva los datos observacionales son aquellos que no han sido generados por mecanismos de experimentos aleatorios, donde incluimos encuestas, registros administrativos o datos censales (Winship y Morgan 1999). Es en estudios observacionales que se utiliza el abordaje contrafáctico.

Para Shadish et al., (2001) la mejor manera de entender un efecto es a través del modelo contrafáctico que tiene sus raíces en la filosofía de David Hume en el

${ }^{2}$ El más claro ejemplo de experimentos aleatorios está inspirado en los trabajos de Ronald A.Fisher en los años veinte del siglo XX (Shadish, Cook, y Campbell 2001; S. Y. Guo y Fraser 2010). Para la Real Academia Española, experimentar cuando está referido a las ciencias fisicoquímicas y naturales implica "hacer operaciones destinadas a descubrir, comprobar o demostrar determinados fenómenos o principios científicos." 
siglo XVIII. Lo contrafáctico es algo que es contrario a los hechos. En un experimento, observamos lo que pasó cuando las personas reciben un tratamiento. Lo contrafáctico es el conocimiento de lo que hubiera ocurrido a aquellas mismas personas si no hubieran recibido el tratamiento. El efecto, pues, es la diferencia entre lo que ocurrió y lo que hubiera ocurrido.

Para Morgan y Winship (2007), el núcleo básico del abordaje contrafáctico para el análisis de datos observacionales es simple. Supongamos que cada individuo de la población de interés puede estar expuesto a estados alternativos de una causa. Cada estado está caracterizado por un conjunto distinto de condiciones, que afectará los potenciales resultados de interés, como las ganancias en el mercado de trabajo o la puntuación en un test estandarizado de matemática. Si la variable de estudio son los ingresos, la población de interés podría ser la de adultos entre los 30 y 50 años, y los dos estados podrían ser si el individuo tiene o no título universitario. Si la variable de interés es la puntuación en un test de matemática, la población de interés podría ser la de alumnos del colegio secundario y los dos estados si el estudiante ha tomado cursos de trigonometría o no. Según Morgan y Winship (2007), en la tradición contrafáctica estos estados causales alternativos son referidos como "tratamientos alternativos". Cuando consideramos solo dos tratamientos, estos son referidos como "tratamiento" y "control".

El supuesto fundamental del modelo contrafáctico, para Morgan y Winship (2007), es que cada individuo en la población de interés tiene un potencial resultado bajo cada estado de tratamiento, inclusive cuando los individuos puedan ser observados sólo en un estado en un momento en el tiempo. Por ejemplo, para analizar el efecto causal de tener un grado universitario, los adultos que han completado la universidad tienen un salario "potencial" bajo el estado de haber completado sólo el secundario. Asimismo, los individuos que han completado sólo el secundario tienen teóricamente un ingreso "si hubieran completado" la universidad. Estos estados "potenciales" son "contrafácticos".

Morgan y Winship (2007) formalizan esta conceptualización de un tratamiento y dos estados: los resultados potenciales de cada individuo i son $\mathrm{y}_{\mathrm{i}}{ }^{\mathrm{e}} \mathrm{e}$ $\mathrm{y}_{\mathrm{i}}{ }^{0}$, donde el superíndice 1 indica el estado de tratamiento y el superíndice 0 indica el estado de control.

Ambos estados $y_{i}{ }_{i}^{1}$ e $y_{i}^{0}$ existen en teoría para cada individuo, por lo tanto el efecto causal puede ser definido por algún tipo de contraste entre $y_{i}{ }^{1}$ e $y_{i}{ }^{0}$, generalmente la diferencia $y_{i}{ }^{1}-y_{i}{ }^{0}$. Como es imposible observar ambos estados, los efectos causales no pueden ser calculados a este nivel. Se presenta la siguiente tabla resumen ${ }^{3}$.

${ }^{3}$ Para Morgan y Winship (2007) la única estrategia efectiva para estimar el efecto causal a este nivel es a través de diseños "crossover", en el cuales los individuos están expuestos a dos tratamientos alternativos sucesivos y con el suficiente tiempo entre las exposiciones para que el efecto de la causa pueda disiparse. Obviamente, este diseño solo puede lograrse cuando el investigador tiene el control sobre la asignación de los tratamientos y solo cuando los efectos del tratamiento son lo suficientemente efímeros. Estas condiciones raramente existen para las preguntas de los científicos sociales. 
Tabla 1. Enfoque contrafáctico

\begin{tabular}{|l|l|l|}
\hline Grupo & $\mathbf{Y}^{\mathbf{1}}$ & $\mathbf{Y}^{\mathbf{0}}$ \\
\hline Tratamiento(D=1) & Observable & $($ Contrafáctico $)$ \\
\hline Control $(\mathrm{D}=0)$ & (Contrafáctico) & Observable \\
\hline
\end{tabular}

Fuente: Morgan y Winship (2007)

Siguiendo con el argumento de Morgan y Winship (2007), el investigador debe analizar una variable de resultado $\mathrm{Y}$ que toma los valores de $\mathrm{y}_{\mathrm{i}}$ para cada individuo i que es igual a $y_{i}{ }^{1}$ para aquellos individuos en el estado de tratamiento e $\mathrm{y}_{\mathrm{i}}^{0}$ para aquellos individuos en el estado de control. Generalmente nos referimos al estado de tratamiento como grupo de tratamiento y aquellos en el estado de control como grupo de control. En consecuencia, $y_{\dot{j}}{ }^{\text {es }}$ un resultado contrafáctico inobservable para cada individuo i en el grupo de tratamiento e $\mathrm{y}_{\mathrm{i}}{ }^{1}$ es un resultado contrafáctico inobservable para cada individuo i en el grupo de control.

En la tradición contrafáctica, la atención está en estimar diversos efectos causales promedios, mediante el análisis de los valores de $\mathrm{y}_{\mathrm{i}}$ para grupos de individuos definidos por características específicas. Para hacer esto efectivamente, debe ser modelado el proceso a través del cual los individuos de diferentes tipos están expuestos a la causa de interés. La atención se focaliza en la estimación de varios tipos de efectos promedios, mediante el análisis de los valores de $\mathrm{y}_{\mathrm{i}}$, grupos de individuos definidos por específicas características.

Los desafíos de utilizar datos observacionales para justificar afirmaciones causales son considerables (Winship y Morgan 1999; Shadish, Cook, y Campbell 2001; Goldthorpe 2001; Smith 2003; Winship y Sobel 2009; Rosenbaum 2009; Gangl 2010). En ese sentido esta tradición confía en el uso del lenguaje experimental en las ciencias sociales observacionales.

\section{EL ENFOQUE DE LA PUNTUACIÓN DE LA PROPENSIÓN}

Seguimos los trabajos de Rosenbaum y Rubin $(1983 ; 1985)$ y utilizamos el enfoque de "análisis de la puntuación de la propensión"(propensity score analysis) (Morgan 2001; S. Guo, Barth, y Gibbons 2006; Caliendo y Kopeinig 2008; S. Y. Guo y Fraser 2010; Myers y Thomas 2010; Pattanayak, Rubin, y Zell 2011; Sekhon 2010; Shadish y Steiner 2010; Austin 2011)para solucionar el sesgo de selección inherente cuando analizamos el efecto de las remesas en áreas de origen. Este método es una manera de articular el enfoque contrafáctico con la generación de escenarios concretos que permitan la comparabilidad entre grupos. El método de puntuación de la propensión es un método estadístico relativamente nuevo e innovador que ha probado ser útil en la evaluación de los efectos de algún "tratamiento" cuando se utilizan datos no experimentales, es decir, que provienen de observaciones no controladas (Thoemmes y Kim 2011). 
La motivación del surgimiento de esta técnica proviene de la necesidad de analizar efectos causales de tratamientos en datos observacionales y reducir el sesgo de selección (S. Guo, Barth, y Gibbons 2006). Consideramos que evaluar el impacto de la recepción de remesas sin considerar el problema del sesgo de selección conduce a errores. El análisis de la puntuación de la propensión esencialmente involucra tres pasos (S. Guo, Barth, y Gibbons 2006; S. Y. Guo y Fraser 2010).

El primero es la estimación de la puntuación de la propensión, que requiere que se identifiquen las variables cuyos efectos serán bloqueados en la comparación.

El segundo es el apareo ("matching") de los hogares tratados con los no tratados en función de su puntuación en la propensión. El resultado es una submuestra de la original, por lo que este paso se llama re-muestreo. De este modo, los hogares que reciben remesas son apareados con otros hogares que son similares excepto que no reciben remesas. La literatura reconoce el algoritmo del "vecino más cercano con calibrador" como uno de los más utilizados." (S. Guo, Barth, y Gibbons 2006; S. Y. Guo y Fraser 2010). Para Thoemmes y Kim (2011) existe un debate en el campo de la metodología sobre la mejor manera de evaluar el apareo. Autores como Ho, Imai, King, \& Stuart (2007) critican las pruebas de significación debido a la dependencia del tamaño de la muestra y sugieren examinar las diferencias estandarizadas antes y después del apareo. Esta diferencia estandarizada es una medida muy utilizada en el campo de la Psicología, conocida como "d de Cohen" (Cohen 1988). El argumento es que el uso de las pruebas de significación -como la prueba t-, en algunas circunstancias pueden ser erróneas debido a una baja potencia estadística. El tercer paso es el análisis de los efectos del tratamiento basado en las muestras.

\section{EFECTOS DE LAS REMESAS EN LA ESCOLARIZACIÓN. APLICACIÓN.}

En nuestro trabajo aplicamos los siguientes pasos:

a) Estimamos la probabilidad de recibir remesas del Sur Global (Argentina y Brasil) en hogares paraguayos como función de características observables del hogar y del jefe del hogar. Se establece un ranking de hogares receptores y no receptores en función de su "puntuación de la propensión".

b) Realizamos un matching entre hogares receptores con hogares no receptores en función de similares puntuaciones en su propensión.

c) Calculamos las diferencias en diversos componentes de la escolarización.

Se utiliza el paquete MatchIt (Ho et al. 2007; Ho et al. 2011) del software R. La fuente de datos utilizada es la Encuesta Permanente de Hogares de Paraguay del año 2009. 
El objetivo principal de la encuesta fue generar datos relacionados con el empleo, el desempleo, los ingresos y otras características sociales y económicas, que permitan conocer la evolución del bienestar de la población paraguaya. La encuesta tiene específicamente dos módulos referidos a migración: uno que indaga sobre la migración de paraguayos en los últimos cinco años, y el otro sobre la composición del ingreso de cada miembro del hogar (si recibe ingresos del exterior y el monto). Se investigan además, los ingresos provenientes de la ocupación principal, de la ocupación secundaria y otras ocupaciones, ingresos por alquileres o rentas, por jubilaciones o pensiones, transferencias familiares regulares en dinero provenientes del país, prestaciones por divorcios y otros ingresos. Se incluye también información sobre las horas trabajadas durante la semana y la condición de actividad o inactividad de los miembros del hogar. La captación se efectúa con periodicidad mensual. La encuesta cubre todos los departamentos del país, excluyendo Alto Paraguay y Boquerón, cuyas poblaciones representan menos del $2 \%$ de la población total del país. El tamaño de la muestra es de 4439 hogares y 18419 individuos. Para este trabajo se utiliza el segundo módulo, sobre la composición del ingreso del hogar.

Estamos interesados en comparar dimensiones de la escolarización en hogares no expuestos al tratamiento $\mathrm{T}=0$ con hogares expuestos al tratamiento $\mathrm{T}=1$. Como solo uno de estos dos estados es observado para cada tipo de hogar, se estima el efecto promedio del tratamiento sobre los tratados (Average Treatment on the Treated- ATT) (Cox-Edwards y Rodríguez-Oreggia 2009), es decir, la diferencia en dimensiones de escolarización entre aquellos hogares receptores y aquellos con la misma probabilidad de ser receptores. Como estamos analizando el efecto de las remesas provenientes del Sur no se consideran los hogares receptores de remesas del Norte Global o remesas internas, creemos esto relevante porque de incluir estos hogares en el análisis se podrían introducir sesgos. El objetivo es trabajar específicamente con el efecto de las remesas provenientes del Sur Global en Paraguay.

\subsection{Estimación de la puntuación de la propensión}

Rosenbaum y Rubin (1985) definen la puntuación de la propensión para los sujetos $\mathrm{i}(\mathrm{i}=1, \ldots \ldots, \mathrm{N})$ como la probabilidad condicional de ser asignado a un particular tratamiento $\left(\mathrm{W}_{\mathrm{i}}=1\right)$ versus el no tratamiento $(\mathrm{Wi}=0)$ dada una serie de vectores de covariables observadas xi:

$$
e\left(x_{i}\right)=\operatorname{pr}\left(W_{i}=1 \mid X_{i}=x_{i}\right)
$$

Generalmente este valor es estimado a través de una regresión logística. Rosenbaum y Rubin (1985) sugieren usar el logit de la probabilidad predicha como 
puntuación de la propensión (ej: $q(x)=\log [(1-\hat{e}(x)) / \hat{e}(x)])$, porque la distribución de $\mathrm{q}(\mathrm{x})$ aproxima una distribución normal ${ }^{4}$.

La ventaja de utilizar la propensión de la puntuación para el matching es que el vector $X$ puede incluir muchas covariables y la multidimensionalidad de utilizar tantas covariables se reduce a la puntuación en solo una (S. Guo, Barth, y Gibbons 2006).

Las principales variables incluidas en el modelo de regresión logística se incluyen en la tabla 2. Se presentan los estadísticos descriptivos, el análisis bivariado y el modelo de regresión logística. De los 3499 hogares paraguayos que componen el universo muestral de la EPH2009, el 7.9\% recibe remesas provenientes del Sur Global (de Argentina y Brasil). El análisis bivariado (chi cuadrado y pruebas $t$ de muestras independientes) muestra que la mayoría de las variables acusan diferencias estadísticamente significativas entre los dos grupos antes del matching, indicando que la distribución de las covariables no está lo suficientemente superpuesta entre el grupo de control y el grupo bajo tratamiento en la muestra original.

En la tabla 2 se presenta un resumen de las principales diferencias encontradas entre los hogares receptores de remesas del Sur y aquellos que no. Los hogares receptores difieren del resto de los hogares de la población (aun cuando en el resto de la población no están incluidos los hogares que reciben remesas del Norte o remesas internas).

Antes de analizar el impacto de las remesas en la escolarización es importante detenernos en examinar las caraterísticas familiares que afectarían la decisión para mandar remesas. En función de los resultados precedentes podemos observar cómo los hogares que reciben remesas del Sur presentan particularidades en relación al resto de la población y que no son una muestra aleatoria de la población en áreas de origen. Están autoseleccionados en función de las variables analizadas. Los hogares receptores tienen predominantemente una jefatura de hogar femenina y de mayor edad promedio que el resto de la población. Los efectos en términos de la composición del hogar son claros, hay menor proporción de jefes en unión y una estructura familiar que se caracteriza por la falta de un hogar nuclear completo. La migración de uno o más miembros del hogar provoca una disrupción en la estructura familiar, tanto en términos de la composición demográfica de la misma como de las rearticulaciones que se producen al interior del hogar. Es decir, se observa claramente un drenaje de población en las edades activas. Sumado a esto, los hogares son predominantemente rurales y con niveles de escolarización más bajos que el resto de la población.

Las observaciones precedentes provocan un desbalanceo de las covariables analizadas. La simple comparación de estos hogares con el resto de la población

${ }^{4}$ En la literatura (Rosenbaum y Rubin 1985; S. Guo, Barth, y Gibbons 2006; S. Y. Guo y Fraser 2010)la cantidad $q(x)$ es también denominada puntuación de la propensión, aunque $q(x)$ difiera de $e(x)$ en función de la ecuación precedente. En este trabajo seguimos la convención de referir $e(x)$ como la puntuación de la propensión. El lector debe tener en consideración que una transformación logit de $e(x)$ puede ser usada ya que $q(x)$ tiene propiedades que la hacen más deseable que $e(x)$. 
para evaluar el efecto de las remesas no es algo deseable. En la figura 1 presentamos las covariables que afectan la asignación no aleatoria al "tratamiento".

Para obtener la puntuación de la propensión utilizamos un modelo de regresión logística, se presentan los coeficientes en la tabla 2. Los hogares que reciben remesas de Argentina o de Brasil fueron codificados como 1 y los hogares que no reciben remesas (ni internas ni del norte) fueron codificados como 0 . Se incluyeron variables relativas a las características del jefe de hogar como así también relativas al hogar. Se incluyó en relación al jefe de hogar las siguientes variables: sexo, edad, nivel de instrucción, estado civil e idioma hablado en el hogar.

En relación a las variables del hogar se incluyeron":

- Proporción de miembros menores de 14 años.

- Proporción de miembros en edad económicamente activa (18-64 años).

- Área urbana o rural donde se ubica el hogar.

- Tipo de hogar.

Tabla 2. Estadísticas Descriptivas y Modelo de Regresión Logística para predecir Puntuación de la Propensión

\begin{tabular}{|c|c|c|c|c|c|c|c|c|c|c|}
\hline \multicolumn{2}{|l|}{ Variables } & $\mathbf{N}$ & $\%$ & $\begin{array}{l}\% \\
\text { remesas } \\
\text { Sur }\end{array}$ & Media & $\begin{array}{l}\text { Desv. } \\
\text { Est. }\end{array}$ & gl & $\begin{array}{l}\text { Bivari- } \\
\text { ado. } \\
\chi 2, \mathrm{t}\end{array}$ & $\mathbf{p}$ & $\begin{array}{l}\text { Logística. } \\
\text { B }\end{array}$ \\
\hline \multicolumn{11}{|c|}{ Jefe de Hogar } \\
\hline \multirow{2}{*}{ Sexo } & (Mujer) & 905 & 25.9 & 40.1 & & & 1 & 32 & 0.000 & \\
\hline & Hombre & 2,594 & 74.1 & 59.9 & & & & & & $-0.595^{* * *}$ \\
\hline \multirow[t]{2}{*}{ Edad } & $\begin{array}{l}\text { Sin reme- } \\
\text { sas }\end{array}$ & & & & 46.5 & 15.0 & 3497 & -8.504 & 0.000 & \\
\hline & Sur Global & & & & 54.4 & 14.3 & & & & $0.106 * * *$ \\
\hline \multirow{2}{*}{$\begin{array}{l}\text { Cuadrado } \\
\text { edad }\end{array}$} & $\begin{array}{l}\text { Sin reme- } \\
\text { sas }\end{array}$ & & & & 2385 & 1533 & 3497 & -8.119 & 0.000 & \\
\hline & Sur Global & & & & 3166 & 1574 & & & & $-0.001 * *$ \\
\hline \multirow{2}{*}{$\begin{array}{l}\text { Estado } \\
\text { Civil }\end{array}$} & $\begin{array}{l}\text { (Sin } \\
\text { unión) }\end{array}$ & 862 & 24.6 & 35.0 & & & 1 & 17.465 & 0.000 & \\
\hline & En & 2,637 & 75.4 & 65.0 & & & & & & $0.416^{*}$ \\
\hline \multirow[t]{2}{*}{$\begin{array}{l}\text { Años de } \\
\text { studio }\end{array}$} & $\begin{array}{l}\text { Sin reme- } \\
\text { sas }\end{array}$ & & & & 7.6 & 4.5 & 380 & 15 & .000 & \\
\hline & Sur global & & & & 4.5 & 3.1 & & & & $-0.115 * * *$ \\
\hline
\end{tabular}

${ }^{5}$ Las variables, proporción de menores de 14 años y proporción entre 18 y 64 años, están dada por el cociente entre la cantidad de personas en esos grupos etarios sobre la cantidad de miembros en el hogar. 


\begin{tabular}{|c|c|c|c|c|c|c|c|c|c|c|}
\hline \multicolumn{2}{|l|}{ Variables } & $\mathbf{N}$ & $\%$ & $\begin{array}{l}\% \\
\text { remesas } \\
\text { Sur }\end{array}$ & Media & $\begin{array}{l}\text { Desv. } \\
\text { Est. }\end{array}$ & gl & $\begin{array}{l}\text { Bivari- } \\
\text { ado. } \\
\chi^{2}, \mathrm{t}\end{array}$ & $\mathbf{p}$ & $\begin{array}{l}\text { Logística. } \\
\text { B }\end{array}$ \\
\hline \multirow{2}{*}{$\begin{array}{l}\text { Idioma en } \\
\text { el Hogar }\end{array}$} & (Otros) & 1,831 & 52.3 & 30.7 & & & 1 & 56 & 0.000 & \\
\hline & $\begin{array}{l}\text { Solo } \\
\text { Guaraní }\end{array}$ & 1,668 & 47.7 & 69.3 & & & & & & 0.294 \\
\hline \multicolumn{11}{|l|}{ Hogar } \\
\hline \multirow[t]{2}{*}{ Prop.$<14$} & $\begin{array}{l}\text { Sin Reme- } \\
\text { sas }\end{array}$ & & & & .264 & .231 & 3497 & -1.455 & .146 & \\
\hline & Sur Global & & & & .285 & .227 & & & & $0.856^{*}$ \\
\hline \multirow[t]{2}{*}{ Prop.18-64 } & $\begin{array}{l}\text { Sin Reme- } \\
\text { sas }\end{array}$ & & & & .604 & .267 & 3497 & 5 & .000 & \\
\hline & Sur Global & & & & .521 & .266 & & & & -0.222 \\
\hline \multirow{6}{*}{$\begin{array}{l}\text { Tipo de } \\
\text { Hogar }\end{array}$} & (Otros) & 1,649 & 47.1 & 76.2 & & & 1 & 102 & 0.000 & \\
\hline & Completo & 1850 & 52.9 & 23.8 & & & & & & $-1.149 * * *$ \\
\hline & (Otros) & 2,128 & 60.8 & 32.5 & & & 1 & 101 & 0.00 & \\
\hline & $\begin{array}{l}\text { Extendido } \\
\text { o inc. }\end{array}$ & 1,371 & 39.2 & 67.5 & & & & & & 0.024 \\
\hline & (Otros) & 3,221 & 92.1 & 91.3 & & & 1 & 0.213 & 0.645 & \\
\hline & $\begin{array}{l}\text { Uniper- } \\
\text { sonal }\end{array}$ & 278 & 8.0 & 8.7 & & & & & & $\mathrm{n} / \mathrm{a}$ \\
\hline \multirow[t]{2}{*}{ Área } & (Rural) & 1,550 & 44.3 & 62.1 & & & 1 & 39 & 0.000 & \\
\hline & Urbana & 1,949 & 55.7 & 37.9 & & & & & & $-0.464 * *$ \\
\hline $\begin{array}{l}\text { Cons- } \\
\text { tante de } \\
\text { regresión } \\
\text { logística }\end{array}$ & & & & & & & & & & $-4.488 * * *$ \\
\hline
\end{tabular}

Categoría de referencia entre parentesis. $* \mathrm{p}<0.05 ; * * \mathrm{p}<0.01 ; * * * \mathrm{p}<0.001$.

Fuente: Elaboración propia en base a Encuesta permanente de hogares, 2009. 


\section{FIGURA 1: desbalanceo de covariables resultado de la asignación al tratamiento de manera no aleatoria para hogares receptores y no receptores}

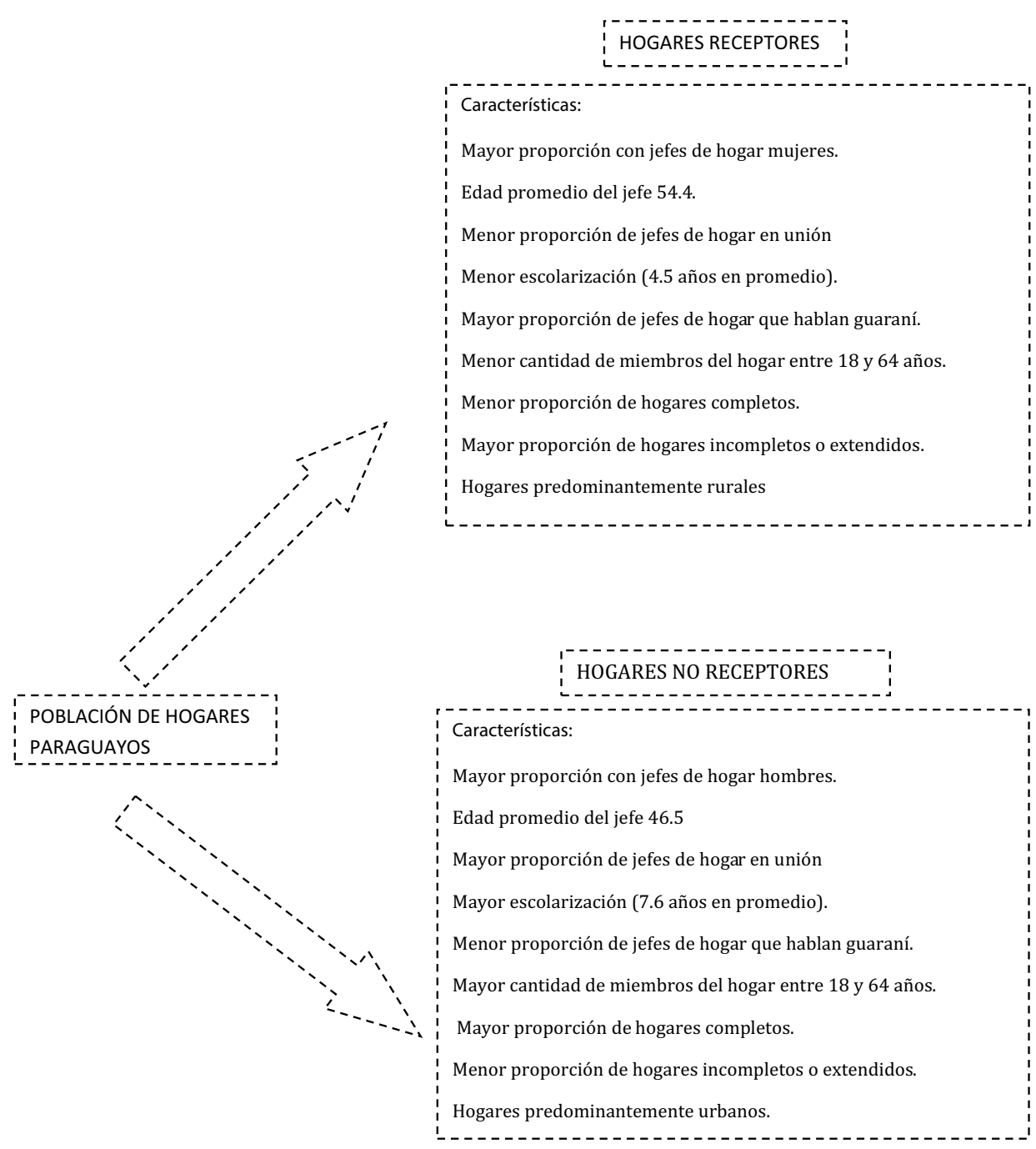

EMPIRIA. Revista de Metodología de Ciencias Sociales. N. ${ }^{\circ}$ 28, mayo-agosto, 2014, pp. 107-128. ISSN: 1139-5737, DOI: 10.5944/empiria.28.12123 


\subsection{Matching o remuestreo}

Después de estimar la puntuación de la propensión realizamos el matching de casos para crear una nueva muestra de casos que comparten aproximadamente la misma probabilidad de ser asignados a la condición de tratamiento. Utilizamos la técnica de apareamiento de vecino más cercado con calibrador. Este método es una combinación de dos abordajes. Se comienza ordenando aleatoriamente los participantes tratados y de control. Se seleccionada el primer participantes tratado $i$ y después se encuentra $j$ como apareo para $i$, si la diferencia absoluta de la puntuación de la propensión entre $i$ y $j$ cae dentro de un calibrador $\varepsilon$ determinado, y si el menor de todos los pares de las diferencias absolutas de la puntuación de la propensión entre $i$ y los otros $j$ está dentro del calibrador. Ambos, $i$ y $j$ son posteriormente removidos de consideración para el próximo apareo y el siguientes participante tratado es seleccionado. El tamaño del calibrador es determinado por el investigador pero en la literatura se sugiere que el tamaño del calibrador sea un cuarto de la desviación estándar de $q(x)$ (Rosenbaum y Rubin 1985); equivalente a $0.25^{*} \sigma q$. El tamaño de las nuevas muestras se presenta en la tabla 3 .

Tabla 3. Tamaño de las nuevas muestras

\begin{tabular}{|l|c|c|}
\hline \multirow{2}{*}{$\begin{array}{l}\text { Descripción del esquema matching y } \\
\text { tamaño de las nuevas muestras }\end{array}$} & \multicolumn{2}{|c|}{ N de las nuevas muestras } \\
\cline { 2 - 3 } & Tratamiento & Control \\
\hline $\begin{array}{l}\text { Puntuación de la propensión basada en } \\
\text { regresión logística, matching uno a uno } \\
\begin{array}{l}\text { a través de vecino más cercano con cali- } \\
\text { brador }^{6}\end{array}\end{array}$ & 277 & 277 \\
\hline
\end{tabular}

Fuente: elaboración propia en base a EPH2006. DGEEC.Paraguay

El objetivo del matching es crear datos que se acerquen lo más posible a un experimento aleatorio (Ho et al. 2011). Cuando se alcanza este objetivo se intenta romper el vínculo entre la variable de tratamiento y la auto-selectividad. En ese punto, es necesario que la distribución de las covariables sea la misma entre el grupo de control y el grupo tratado. Como señalan Ho, Imai, King, \& Stuart (2011), un elemento crucial es evaluar la distribución empírica de las covariables en los dos grupos creados: el "balanceo". Seguimos la recomendación de Ho, Imai, King, \& Stuart (2011) y examinamos las diferencias medias estandarizadas antes y después del matching. Como puede observarse en el gráfico 1, la magnitud de la diferencias después del matching es menor en las nuevas muestras. Esto es un indicador del éxito del re-muestro.

\footnotetext{
${ }^{6}$ De tamaño 0.245 , equivalente a $0.25 * \sigma q$
} 
Gráfico 1: Diferencias de medias estandarizadas (d de cohen). Antes y después del matching

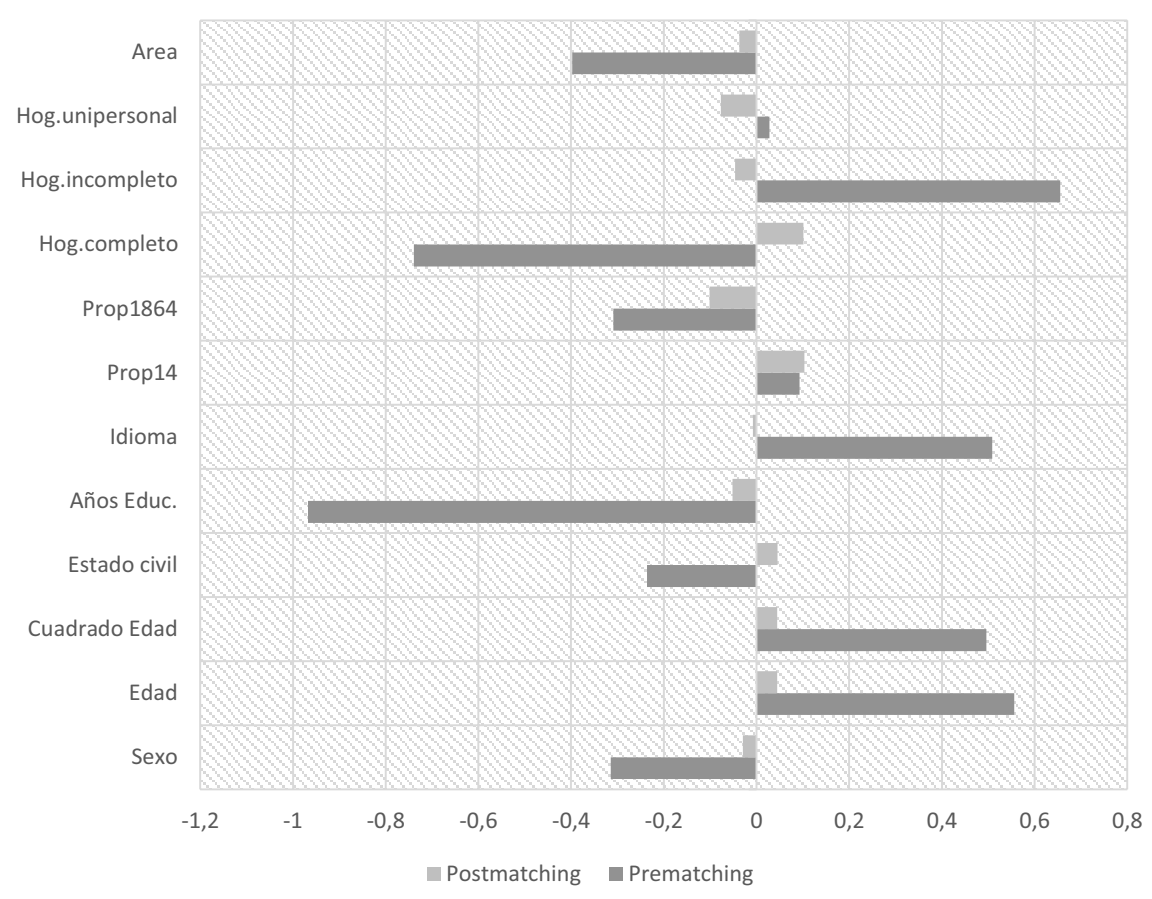

Fuente: elaboración propia en base a EPH2006. DGEEC.Paraguay.

\subsection{Análisis bivariado basado en la nueva muestra}

Con la nueva muestra derivada del proceso de apareo (matching), obtenemos grupos comparables o balanceados en relación a la puntuación de la propensión en el tratamiento. Así, puede ser utilizada la variable dicotómica que indica la pertenencia al grupo receptor de remesas (grupo tratado) o no receptor (no tratado) para realizar comparaciones entre diferentes variables. Los resultados de presentan en la tabla 4. Se analizan dos indicadores: a) la tasa neta de escolarización, definida como el cociente entre personas con edad x matriculadas o siguiendo algún tipo de estudios y el total de la población de esa edad y b) el gasto realizado por el hogar en variables relacionadas con la educación y la formación de capital humano. De los indicadores analizados no encontramos diferencias significativas en ningún componente de la escolarización. 
Tabla 4.Efectos de las remesas en los componentes de la escolarización

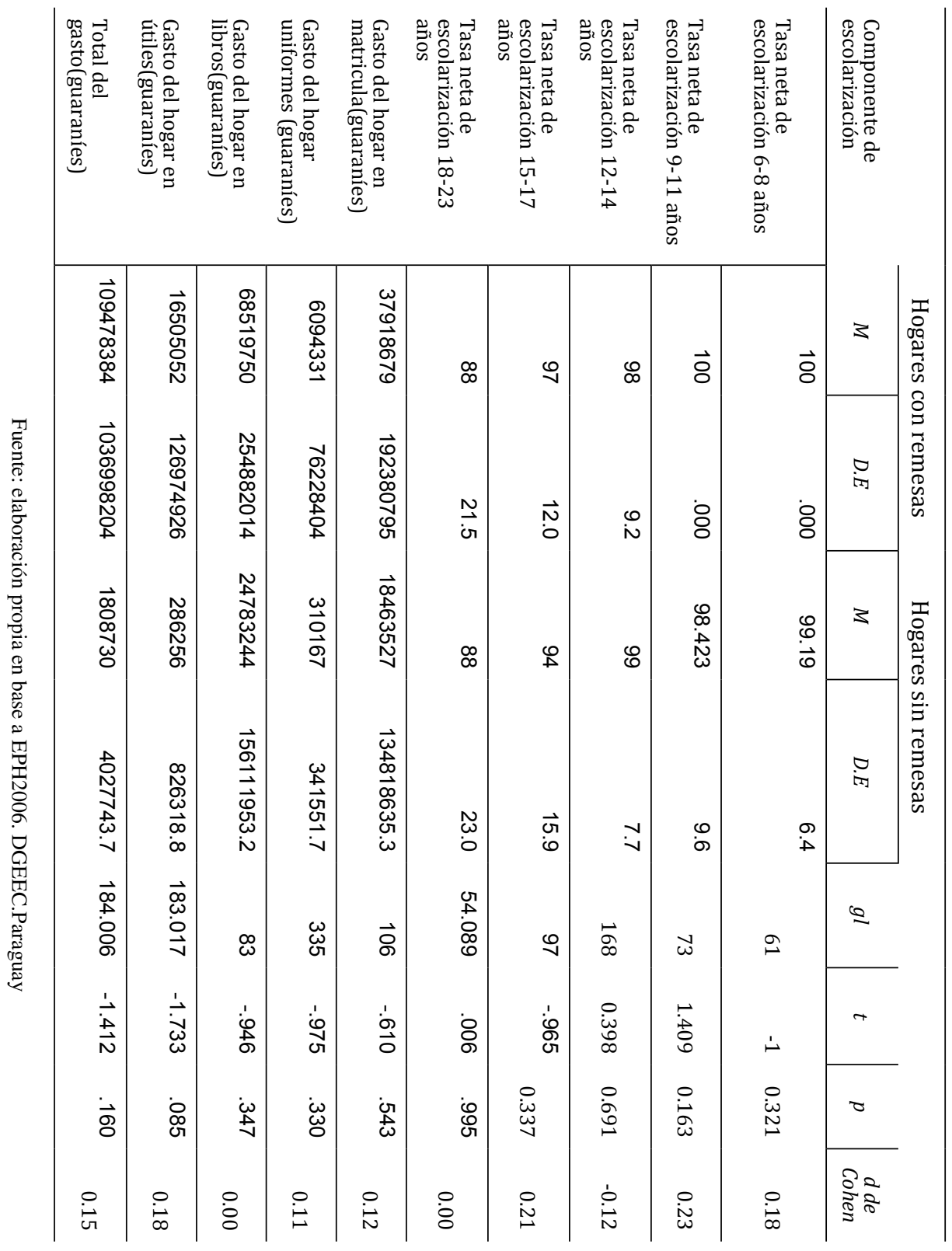




\section{DISCUSIÓN}

Las remesas en Paraguay están vínculadas a dos macro-sistemas migratorios, provienen de Argentina y Brasil en el Sur Global y España y Estados Unidos en el Norte Global. En Argentina, principal destino migratorio, los hombres se insertan mayoritariamente en el sector de la construcción y las mujeres en el trabajo doméstico (Bruno 2013). Cerruti y Parrado (2003) señalan que la migración paraguaya hacia la Argentina está relacionada con las características individuales, la extensión de las redes sociales y la experiencia, pero también con los cambios en las condiciones macroeconómicas. Los resultados de su estudio muestran que el flujo migratorio es muy sensible a las fluctuaciones en las condiciones macroeconómicas, particularmente los diferenciales de ingresos y los cambios en la valuación del peso argentino. De manera específica, los hogares receptores de remesas presentan particularidades en cuanto al lugar que ocupan en la estructura social, aquellos vínculados al Norte Global presentan mayores niveles de escolarización y mejor nivel socioeconómico que los vinculados al Sur Global (Gómez 2013a). Las remesas al interior de la economía doméstica constituyen un elemento fundamental de la composición del ingreso, señalando la alta dependencia del flujo monetario proveniente del exterior. Sin embargo, este peso varía según la procedencia, es mayor en la economia de lo hogares vínculados al Norte (Gómez 2013a).

A escala global, la literatura examina si las remesas tienen impacto en la inversión en capital humano en los países de origen, particularmente en salud y escolarización (Kanaiaupuni y Donato 1999; Cox-Edwards y Ureta 2003; Hildebrandt y McKenzie 2005; Yang y Martínez 2006; Medina y Cardona 2010; Acosta 2011). Existe cierto consenso en torno a que las remesas provenientes de las migraciones internacionales pueden conducir a la formación de capital humano en lugares de origen de la migración. Sin embargo, analizar el impacto de las remesas en la salud o la educación es complejo porque la migración en sí es una variable de elección y endógena (McKenzie y Sasin 2007). Como pudimos analizar en nuestro caso, los resultados obtenidos sugieren que las remesas provenientes del Sur Global en Paraguay no pueden traducirse en formación de capital humano a través de la capitalización en diversas dimensiones de la escolarización. El contexto de estos hogares presenta una doble característica: a) la inserción precaria de los migrantes en el mercado laboral Argentino (Bruno 2013) y la ubicación de los hogares receptores en el segmento más informal y fragil del mercado de trabajo paraguayo (Gómez y Bologna 2013).

En función de nuestros hallazgos, en las áreas receptoras, la evidencia empírica sugiere tres procesos convergentes: 1) la disminución de la participación laboral en los hogares receptores ; 2) la fragilidad de estos hogares en el mercado laboral (Gómez y Bologna 2013); 3) la dificultad para la formación de capital humano, debido a las características de vulnerabilidad de los hogares receptores.

La inversión de las remesas en capital humano es una de las claves para promover el desarrollo (Acosta 2011). Los resultados en otros contextos, como el Salvador (Cox-Edwards y Ureta 2003), sugieren que hay un impacto significa- 
tivo en la escolarización de los niños. Sin embargo, no hay evidencia que sugiera ese proceso en Paraguay.

Las remesas internacionales son variables endógenas, cuyas funciones sociales oscilan entre la inversión productiva y la simple reproducción de la unidad doméstica (Gómez 2013b). La evidencia empíria sugiere que en el caso analizado, la dimensión privilegiada es la reproducción de la unidad doméstica y no la transformación de este flujo de dinero en algún tipo de inversión productiva. Es decir, la recepción de remesas no es una variable independiente que se reproduce a si misma o un dato exógeno dado, que afecta el desarrollo (de manera equivalente a una política pública); por el contrario, es una variable dependiente y endógena de una dinámica de desarrollo desigual.

\section{CONCLUSIONES}

Los estudios sobre el impacto de las remesas no son conclusivos al respecto. En efecto, existe una heterogeneidad sumamente amplia de conclusiones, tanto en función de los instrumentos utilizados como de las unidades de análisis bajo consideración y los marcos analíticos. El objetivo de este trabajo fue presentar el abordaje contrafáctico como opción metodológica y evaluar los efectos de las remesas internacionales en diversos componentes de la escolarización en Paraguay. Uno de los principales problemas que se enfrenta es el de la posibilidad de comparación entre conjunto de hogares receptores y no-receptores. Problema compartido por gran parte de la investigación en ciencias sociales que trabaja en términos de inferencia causal.

En el artículo se presentaron los problemas derivados de trabajar con la inferencia causal, el abordaje contráfactico en su dimensión conceptual y su aplicación práctica a través del software R y el paquete MatchIt (Ho et al. 2007; Ho et al. 2011). Se consideró al hogar como unidad de analisis y para lograr precisión se trabajaron en detalle los problemas que se derivan cuando evaluamos el impacto de las remesas. Esto es importante porque la intrínseca endogeneidad y selectividad migratoria se convierten en un obstáculo para la precisa evaluación. Los resultados obtenidos sugieren que las remesas provenientes del Sur en Paraguay no tienen efecto en la inversión en capital humano. La metodología propuesta, como es de suponer, puede utilizarse en la evaluación del impacto de diferentes variables de interés. Impacto que estudiamos en hogares y no individuos, que articulan sus decisiones y estrategias de vida en el marco de una estructura económica, social y política como la paraguaya. La metodología propuesta agrega precisión al análisis, pues de no considerar los problemas mencionados se estaría arribando a conclusiones sesgadas e imprecisas. Este tipo de conclusiones sesgadas conspira contra la construcción de teoría correctamente fundada. 


\section{BIBLIOGRAFÍA}

ACOSTA, P. (2011): "School Attendance, Child Labour, and Remittances from International Migration in El Salvador", Journal of Development Studies, 47(6), pp. 913936.

AKEE, R. (2010): "Who Leaves? Deciphering Immigrant Self-Selection from a Developing Country", Economic Development and Cultural Change, 58(2), pp. 323-344.

AUSTIN, P. C. (2011): "Optimal caliper widths for propensity-score matching when estimating differences in means and differences in proportions in observational studies", Pharmaceutical Statistics, 10(2), pp. 150-161.

BERTOLI, S. (2010): "The informational structure of migration decision and migrants self-selection", Economics Letters, 108(1), pp. 89-92.

BORJAS, G. J. (1987): "Self-Selection and the Earnings of Immigrants", The American Economic Review, 77(4), pp. 531-553.

BORJAS, G. J. (1990): "Self-Selection and the Earnings of Immigrants: Reply", The American Economic Review, 80(1), pp. 305-308

BRUNO, S.; (2013): "El proceso migratorio paraguayo hacia Argentina: evolución histórica, dinámica asociativa y caracterización sociodemográfica y laboral", en Migrantes paraguayos en Argentina: Población, instituciones y discursos, Buenos Aires, OIM, pp.11-47.

CALIENDO, M.; KOPEINIG, S. (2008): "Some Practical Guidance for the Implementation of Propensity Score Matching", Journal of Economic Surveys, 22(1), pp. 3172.

CHIQUIAR, D.; HANSON, G. H. (2005): "International Migration, Self-Selection, and the Distribution of Wages: Evidence from Mexico and the United States", The Journal of Political Economy, 113(2), pp. 239-281.

CHISWICK, B. (1999): “Are Immigrants Favorably Self-Selected?”, American Economic Review, 89(2), pp. 181-185

COCHRAN, W. G. (1965): "The Planning of Observational Studies of Human Populations", Journal of the Royal Statistical Society. Series A (General), 128(2), pp. 234-266.

COHEN, J. (1988): Statistical Power Analysis for the Behavioral Sciences (2.a ed.), Estados Unidos, Routledge Academic.

CONSTANT, A.; MASSEY, D. S. (2003): "Self-Selection, Earnings, and Out-Migration: A Longitudinal Study of Immigrants to Germany", Journal of Population Economics, 16(4), pp. 631-653.

COULON, A. de; PIRACHA, M. (2005) : "Self-Selection and the Performance of Return Migrants: The Source Country Perspective", Journal of Population Economics, 18(4), pp. 779-807.

COX-EDWARDS, A.; RODRÍGUEZ-OREGGIA, E. (2009): "Remittances and Labor Force Participation in Mexico: An Analysis Using Propensity Score Matching", World Development, 37(5), pp. 1004-1014.

COX-EDWARDS, A.; URETA, M. (2003): "International migration, remittances, and schooling: evidence from El Salvador", Journal of Development Economics, 72(2), pp. 429-461.

DGEEC Paraguay. (2009). DGEEC. Encuesta Permanente de Hogares 2009 .Dirección General de Estadística, Encuestas y Censos. Secretaría Técnica de Planificación de la Presidencia de la República, Asunción, Paraguay. 
FUNKHOUSER, E. (2006): "The Effect of Emigration on the Labor Market Outcomes of Sender Households: A Longitudinal Approach Using Data from Nicaragua", Well-Being and Social Policy, 2(2), pp. 5-25.

GANGL, M. (2010): "Causal Inference in Sociological Research", Annual Review of Sociology, 36(1), pp. 21-47.

GÓMEZ, P.S.;(2013a): "Remesas y estratificación social en Paraguay: dinámica de la recepción del remesas del Sur y del Norte", Migraciones, 37, pp.97-125

GÓMEZ, P.S.;(2013b): "Migraciones, remesas internacionales y desarrollo en el cono sur de América Latina: perspectivas analíticas", Estudios, 30,pp.90-110.

GÓMEZ, P.S.; BOLOGNA, E.; (2013): "Remesas y participación laboral en paraguay: efectos de los desplazamientos sur-sur", Migraciones internacionales, 25(2), pp.189218.

GOLDTHORPE, J. H.; (2001): “Causation, Statistics, and Sociology”, European Sociological Review, 17(1), pp. $1-20$.

GUO, S.; BARTH, R. P.; GIBBONS, C. (2006): "Propensity score matching strategies for evaluating substance abuse services for child welfare clients", Children and Youth Services Review, 28(4), pp. 357-383.

GUO, S. Y.; FRASER, M. W. (2010): Propensity Score Analysis: Statistical Methods and Applications,

(1.a ed.), Thousand Oaks, Estados Unidos, Sage Publications.

HECKMAN, J.; (1979): "Sample Selection Bias as a Specification Error”, Econometrica, 47 (1), pp. 153-161

HILDEBRANDT, N.; McKENZIE, D. J. (2005): “The Effects of Migration on Child Health in Mexico", Economía, 6(1), pp. 257-289.

HO, D.; KOSUKE, I.; KING, G.; STUART, E. (2007): "Matching as Nonparametric Preprocessing for Reducing Model Dependence in Parametric Causal Inference", Political Analysis, 15(3), pp. 199-236.

HO, D.; KOSUKE, I.; KING, G.; STUART, E. (2011): "Matchit: Nonparametric Preprocessing for Parametric Causal Inference", Journal of Statistical Software, 42 (8), p. $1-28$.

KANAIAUPUNI, S. M.; DONATO, K. M. (1999): "Migradollars and Mortality: The Effects of Migration on Infant Survival in Mexico", Demography, 36(3), pp. 339353.

McKENZIE, D.; SASIN, M. (2007): "Migration, remittances, poverty, and human capital : conceptual and empirical challenges". Policy Research Working Paper Series 4272. The World Bank, pp.1-16.

McKENZIE, D.; STILLMAN, S.; GIBSON, J. (2010): "How Important is Selection? Experimental VS. Non-Experimental Measures of the Income Gains from Migration", Journal of the European Economic Association, 8(4), pp. 913-945.

MEDINA, C.; CARDONA, L. (2010): "The Effects of Remittances on Household Consumption, Education Attendance and Living Standards: the Case of Colombia", Lecturas de Economía, 72(72), pp. 11-44.

MORGAN, S. L. (2001): "Counterfactuals, Causal Effect Heterogeneity, and the Catholic School Effect on Learning", Sociology of Education, 74(4), pp. 341-374.

MORGAN, S. L.; WINSHIP, C. (2007): Counterfactuals and Causal Inference: Methods and Principles for Social Research (1.a ed.). Cambridge University Press.

MYERS, J. ; THOMAS, L. A. (2010): "Regression Adjustment and Stratification by Propensity Score in Treatment Effect Estimation”, Johns Hopkins University, Dept. 
of Biostatistics Working Papers. Working Paper 203, disponible en http://biostats. bepress.com/jhubiostat/paper203 [consulta: 20-3-2012]

NICHOLS, A. (2007): "Causal inference with observational data", Stata Journal, 7(4), pp. 507-541.

PARRADO, E.; CERRUTTI, M. (2003): "Labor Migration between Developing Countries: The Case of Paraguay and Argentina”, International Migration Review, 37 (1), pp 101-132

PATTANAYAK, C. W.; RUBIN, D. B.; ZELL, E. R. (2011): "Métodos de puntuación de propensión para crear una distribución equilibrada de las covariables en los estudios observacionales", Revista Española de Cardiología, 64(10), pp. 897-903.

ROSENBAUM, P. (2005): "Observational Study", Encyclopedia of Statistics in Behavioral Science. John Wiley \& Sons.

ROSENBAUM, P. R.; RUBIN, D. B. (1983): "The Central Role of the Propensity Score in Observational Studies for Causal Effects", Biometrika, 70(1), pp. 41-55.

ROSENBAUM, P. R.; RUBIN, D. B. (1985): "Constructing a Control Group Using Multivariate Matched Sampling Methods That Incorporate the Propensity Score", The American Statistician, 39(1), pp. 33-38.

ROSENBAUM, P.R. (2009): "Design of Observational Studies",New York, Springer.

SEKHON, J. (2010): "The Neyman-Rubin Model of Causal Inference and Estimation via Matching Methods" en The Oxford Handbook of Political Methodology, Great Britain, Oxford University Press, 271-300.

SHADISH, W. R.; COOK, T. D.; CAMPBELL, D. T. (2001): Experimental and QuasiExperimental Designs for Generalized Causal Inference (2.a ed.), Boston, Houghton Mifflin.

SHADISH, W. R.; STEINER, P. M. (2010): “A Primer on Propensity Score Analysis", Newborn and Infant Nursing Reviews, 10(1), pp. 19-26.

SMITH, H. L. (2003): "Some Thoughts on Causation as It Relates to Demography and Population Studies", Population and Development Review, 29(3), pp. 459-469.

THOEMMES, F. J.; KIM, E. S. A. (2011): "Systematic Review of Propensity Score Methods in the Social Sciences", Multivariate Behavioral Research, v. 46, n. 1, p. 90-118.

TITUS, M. A. (2007): "Detecting Selection Bias, Using Propensity Score Matching, and Estimating Treatment Effect: An Application to the Private Returns to a Master's Degree", Research in Higher Education, 48(4), pp. 487-52.

WEISBERG, H. I. (2010): Bias and Causation: Models and Judgment for Valid Comparisons (1. Ed.), New Jersey, John Wiley \& Sons.

WINSHIP, C.; MORGAN, S. L. (1999): "The Estimation of Causal Effects from Observational Data", Annual Review of Sociology, 25, pp. 659-706.

WINSHIP, C.; SOBEL, M. (2009): "Causal Inference in Sociological Studies", en Handbook of Data Analysis, Londres, Sage Publications Ltd.

YANG, D. C.; MARTINEZ, C. (2006): "Remittances and Poverty in Migrants' Home Areas: Evidence from the Philippines", International Migration, Remittances, and the Brain Drain. Washington, D.C:: World Bank Publications 